\title{
$T=0$ Phase Diagram of the Double-Exchange Model
}

\author{
A. Chattopadhyay ${ }^{1}$, A. J. Millis ${ }^{2}$ and S. Das Sarma ${ }^{1}$ \\ ${ }^{1}$ Department of Physics and MRSEC, University of Maryland \\ College Park, MD 20742 \\ ${ }^{2}$ Center for Materials Theory \\ Department of Physics and Astronomy, Rutgers University \\ Piscataway, NJ 08854
}

(November 16, 2018)

\begin{abstract}
We present the $T=0$ phase diagram of the double-exchange model (ferromagnetic Kondo lattice model) for all values of the carrier concentration $n$ and Hund's couplng $J$, within dynamical mean field theory. We find that depending on the values of $n$ and $J$, the ground state is either a ferromagnet, a commensurate antiferromagnet or some other incommensurate phase with intermediate wave vectors. The antiferromagnetic phase is separated by first order phase boundaries and wide regimes of phase separation. The transition from the ferromagnetic phase to an incommensurate phase is second order.
\end{abstract}

\section{INTRODUCTION}

In this Brief Report we present the $T=0$ magnetic phase diagram of the double exchange modell believed relevant to the physics of 'colossal' magnetoresistance manganites 2 . Our results may also bef interest in the context of magnetic semiconductors 3 . We obtain the different magnetic phases, the boundaries between them, and the regimes of phase separation $u$ sing the dynamical mean field approximation (DMFT) the double-exchange interaction, and study only magnetic ordering, not charge or orbital orfering. This paper is a companion to a previous paper 5 which reported results mainly in regimes believed directly relevant to experiment on manganites. Here we present the complete $T=0$ phase diagram of the model, for the convenience of other workers, and to provide a basis for quantitative comparison of the dynamical mean field method to other methods, i.e the phase diagram given in this paper is not just limited to CMR physics in manganites, but is of much more general validity covering other systems like Mn doped GaAst.

The double-exchange-only (DE) model involves electrons moving in a band structure defined by a hopping matrix $t_{i j}^{a b}$ and a chemical potential $\mu$, and connected by a Hund's coupling $(J>0)$ to core spins $\mathbf{S}$. We denote the operator creating an electron in orbital $a$ with spin $\alpha$ on site $i$ by $d_{i a \alpha}^{\dagger}$ and define the double-exchange only Hamiltonian $H_{D E}$ by

$$
\begin{aligned}
H_{D E}= & -\sum_{<i j>\alpha} t_{i j}^{a b} d_{i a \alpha}^{\dagger} d_{j b \alpha}-\mu \sum_{i \alpha} d_{i \alpha}^{\dagger} d_{i \alpha} \\
& -J \sum_{i \alpha \beta} \vec{S}_{c, i} \cdot d_{i \alpha}^{\dagger} \vec{\sigma}_{\alpha \beta} d_{i \beta} .
\end{aligned}
$$

where $\langle i j\rangle$ implies sum over nearest neighbors.

Note that there are only two independent dimensionless parameters defining $H_{D E}$, since the hopping ampli- tude can be used to define the energy unit. Our interest in this paper is the $T=0$ magnetic phase diagram of $H_{D E}$ as a function of density $n$ and coupling $J$. To compute this we use the dynamical mean field approximation which becomes exact in the limit of spatial dimensionality $d \rightarrow \infty$ and is believed to be reliable in $d=3$ [Ref. 9]. The complete $T=0$ phase diagram of this important model has not to ouf knowledge been presented in any limit. Other worker $\$ 10$ have considered two phases: a commensurate antiferromagnet (AF) and a homogeneous ferromagnet (FM). In a previous paper we demonstrated the existence of a free energy minimum with spin order which is neither AF nor FM; this possible phase has magnetic correlations incommensurate with the underlying lattice and was denoted IC. However, in our previous work the precise spin correlations were not established, nor was the issue of phase separation addressed. The importance of phase separation in the CMR context has been stressed by other workers 11.12. Here, we resolve these issues. As results obtained by other methods become more available, comparison to our results will allow the accuracy of DMFT in $d=3$ to be assessed.

For our specific computations we take the core spins to be classical. This is reasonable because we consider $T=0$ energetics in high spatial dimension, and in cases of physical relevance the core spin is not small $(S=3 / 2$ for the manganites). We proceed by calculating the energies of the different phases. We identify the ground state as the phase of lowest energy and use an explicit Maxwell construction to determine regions of phase separation.

We now discuss the possible phases which may occur. In the $d \rightarrow \infty$ limit, Muller-Hartmann 13 pointed out that the susceptibility depends on the one-dimensional parameter $X(k)=\frac{1}{d} \sum_{i} \cos \left(k_{i}\right)$; similar considerations imply the ordering pattern and the energy are also functions of $X$. Because $-1<X<1$, we write $X=\cos (\theta) ; \theta=0$ corresponds to ferromagnetic (FM) phase and $\theta=\pi$ to antiferromagnetic (AFM) phase considered previously 
$0<\theta<\pi$ coresponds to a phase with incommensurate (IC) spin correlatipns. The specific IC phase treated in our previous work $\mathrm{l}$ corresponds to $\theta=\pi / 2$.

The DMFT solution of Eq. 1 may be written in terms of a spin-dependent mean field function $\mathbf{a}(\omega)$, which obeys an equation that depends on the density of states of the underlying lattice. We study the semicircular density of states $N(\epsilon)=\sqrt{4 t^{2}-\epsilon^{2}} /\left(2 \pi t^{2}\right)$, corresponding to the $d \rightarrow \infty$ limit of the Bethe lattice with nearest neighbor hopping, so that $\mathbf{a}_{i}(\omega)=\omega+\mu-\sum_{\delta} t_{\delta} G_{i+\delta, i+\delta}^{l o c} t_{\delta}$ with $t_{\delta}$ the hopping. To solve the equations, we adopt a space dependent spin basis, quantized parallel to the core spin on site $i$. The conventions just described then imply that a has two independent components $a_{\uparrow}, a_{\downarrow}$ given by

$$
\begin{aligned}
& a_{\uparrow}(\omega)=\omega+\mu-t^{2} \frac{\cos ^{2}(\theta / 2)}{a_{\uparrow}(\omega)+J}-t^{2} \frac{\sin ^{2}(\theta / 2)}{a_{\downarrow}(\omega)-J} \\
& a_{\downarrow}(\omega)=\omega+\mu-t^{2} \frac{\cos ^{2}(\theta / 2)}{a_{\downarrow}(\omega)-J}-t^{2} \frac{\sin ^{2}(\theta / 2)}{a_{\uparrow}(\omega)+J}
\end{aligned}
$$

These equations contain as special cases the equations previously studied for the FM, AFM and $\theta=\pi / 2$ phases. They may be obtained by minimizing with respect to $a_{\uparrow}, a_{\downarrow}$, the energy

$$
\begin{array}{r}
E=T \sum_{n} \frac{\left[a\left(\omega_{n}\right)-\left(\omega_{n}+\mu\right)\right]^{2}}{t^{2}}+\frac{b\left(\omega_{n}\right)^{2}}{t^{2} \cos \theta} \\
+\ln \left[a\left(\omega_{n}\right)^{2}-\left(b\left(\omega_{n}\right)+J\right)^{2}\right]
\end{array}
$$

where $a=\left(a_{\uparrow}+a_{\downarrow}\right) / 2$ and $b=\left(a_{\uparrow}-a_{\downarrow}\right) / 2$. This equation may be transformed into the familiar expression 14

$$
\begin{aligned}
E & =\int d \epsilon_{k} N\left(\epsilon_{k}\right) \int_{-\infty}^{\infty} \frac{d \omega}{\pi} f(\omega) \omega \operatorname{Tr}\left[A\left(\epsilon_{k}, \omega\right)\right] \\
& =\int_{-\infty}^{\infty} \frac{d \omega}{\pi} f(\omega) \omega \operatorname{Tr}\left[\operatorname{Im} G_{l o c}(\omega)\right]
\end{aligned}
$$

through an integration by parts and use of the mean field equations (recalling that $E$ is extremized by $a_{\uparrow}, a_{\downarrow}$ ). Here $N\left(\epsilon_{k}\right)$ is the "band" density of states determined from the hopping $t_{i j}^{a b}, A\left(\epsilon_{k}, \omega\right)=-\frac{1}{\pi} \operatorname{Im} G\left(\epsilon_{k}, \omega\right)$ and the electron Green function $\mathbf{G}=\left(\omega+\mu-\epsilon_{k}-\boldsymbol{\Sigma}(\omega)\right)^{-1} \cdot \boldsymbol{\Sigma}=\mathbf{a}-\mathbf{G}_{l o c}^{-1}$ is the self energy, which is local (k-independent) but dependent on spin in the dynamical mean field approximation.

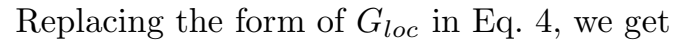

$$
E=\int \frac{d \omega}{\pi} f(\omega) \omega \operatorname{Im}\left(\frac{2 a(\omega)}{a(\omega)^{2}-[b(\omega)+J]^{2}}\right)
$$

We have solved the mean field equations (Eq. 2) and computed the energies for a wide range of $\theta, n$. The resulting phase diagram is shown in Fig. 1. As one can see from Fig. 1, a homogeneous ferromagnetic phase is supported over a wide range of fillings and couplings. There is a small island of IC phase characterized by a $\theta$ which varies with position inside the IC phase. The AFM phase exists only at $n=1$. The FM-IC phase boundary is apprently second order; all of the other phase boundaries are (within our numerical accuracy) first-order and are accompanied by regions of phase separation. At sufficiently small $J$, phase separation preempts the IC phase entirely even though this phase minimizes the energy for $0.47 \leq n \leq 0.7$.

Fig. 2 shows energy as a function of $n$ for the FM and $\mathrm{AF}$ states, as well as $\theta=\pi / 2$ and $3 \pi / 4$. We see immediately from these curves that at $n=1$ the $\mathrm{AF}$ phase is the lowest; at intermediate $n$ there is a sequence of IC phases characterized by a varying angle and at small $n$ the FM phase is stable. Between the $n=1$ AF phase and the intermediate $n \mathrm{IC}$ phase there is a region of phase separation, and the $\theta$ value at the PS-IC boundary is a non-universal $J$-dependent number. Fig. 3 shows the behavior in the vicinity of the FM/IC phase boundary. We see that the transition is apprently second-order, with $\theta$ evolving smoothly to 0 at the FM/IC phase boundary.

Fig. A shows the behavior for $J=0.6 t$ and $n$ close to 1. A Maxwell construction shows that the $\theta=7 \pi / 8$ curve lies higher than the line connecting the $\mathrm{AF} n=1$ point and the $\theta=3 \pi / 4, n \approx 0.78$ point. The energy differences involved are seen to be tiny, implying large uncertainties in the location of the phase boundary and rather complicated behavior in physical systems.

A generally noteworthy feature of Fig. 3 is the extremely small energy differences between different phases: typically the energy difference per site $\Delta E$ between the lowest and next lowest phase is only about $0.02 t$. For manganites, a reasonable value of $t$ is $0.65 \mathrm{eV}$ leading to $\Delta E /$ site $\sim 100 K$. This suggests an unusualsensitivity to disorder, as already noted by Moreo et al.1.5.

We now use analytic arguments to analyze more carefully the behavior in the small $J$ limit, where the energy may be written as

$$
E=-\frac{1}{2} \chi(\theta) J^{2}
$$

with

$$
\chi(\theta)=-2 \int \frac{d \omega}{\pi} f(\omega) \operatorname{Im}\left(\frac{1}{a_{0}(\omega)^{2}-t^{2} \cos \theta}\right)
$$

and $a_{0}=\frac{1}{2}\left[\omega+\mu-\sqrt{(\omega+\mu)^{2}-4 t^{2}}\right]$

Note that the behavior as $\theta \rightarrow \pi$ and $n \rightarrow 1$ is complicated because the energy of the $n=1, \theta=\pi$ AFM phase $\sim J^{2} \ln (1 / J)$. From the explicit energy expression we find (neglecting $O\left(J^{4}\right)$ )

$$
\begin{aligned}
\frac{\partial E_{A F}}{\partial J^{2}}= & \frac{J}{4}\left[E\left(\sin ^{-1}(\mu / 2) ;-4 / J^{2}\right)-E\left(\pi / 2 ;-4 / J^{2}\right)\right]- \\
& \frac{4+J^{2}}{4 J}\left[F\left(\sin ^{-1}(\mu / 2) ;-4 / J^{2}\right)-F\left(\pi / 2 ;-4 / J^{2}\right)\right]
\end{aligned}
$$

where $F(\phi ; m), E(\phi ; m)$ are elliptic integrals of the First and Second Kind. The corresponding energies are plotted in Fig. 5 for $J=0.05$. Note that for $n$ very near 1 
$(\mu<J), \chi_{A F}$ is linear in $1-n$. Thus at $n \neq 1$ the AF phase is always unstable to phase separation. The Maxwell construction also shows that in the small $J$ limit the IC phase is preempted entirely, and the ferromagnetic phase is only stable for $n \leq 0.15$.

We now briefly discuss the physical meaning of the IC phase. From Eq. 6, the lowest energy phase is the one with the largest $\chi(\theta)$. For typical band structures even in $d=3 \chi$ takes one value at $q=0$ and another value at $q=Q=(\pi, \pi, \pi)$; the variation between these limits is smooth for momenta along the zone diagonal, but over much of the Brillouin zone $\chi$ is quite weakly momentum dependent. In the $d \rightarrow \infty$ limit this weak momentum dependence disappears: $\chi(\vec{q})$ at any $\vec{q} \neq \lambda(\pi, \pi, \ldots)$ takes the $q$-independent value $\chi_{l o c}=\sum_{q} \chi(\vec{q})$ and along the diagonal. For $d$ large but finite, $\chi$ varies rapidly with $q$ crossing over from its $\vec{q}=\lambda(\pi, \pi, \ldots)$ values to a very weakly $q$-dependent value in a range $\delta q \approx O(1 / \sqrt{d})$. Thus the hallmark of the phases which are characterized by a small $\cos \theta$ is a large range of spin arrangements very nearby in energy. We suspect that this regime would appear experimentally as a spin glass in the presence of disorder

In conclusion, we have determined the $T=0$ phase diagram of the double-exchange Hamiltonian including regions of phase separation, using analytic arguments at small and large $J$ and the dynamical mean field method We find that the incommensurate phase noted in Ref.E has a rather small region of existence, being mostly overwhelmed by phase separation, and have found the differences in energy between the different phases to be very small.

We have learned of related work by L. Yin and J. Ho (APS March Meeting 2000 Bulletin; L. Yin and J. Ho, private communication), who used a different mean-field technique in $d=3$ to obtain a phase diagram including spiral states, with regions of phase separation qualitatively similar to what is found here. Our results are also similar to the phase diagram of Alonso et al. 16 .

Acknowledgements We thank J. K. Freericks for suggesting that we examine the $\theta$ dependence of the incomensurate phase and the University of Maryland NSF-MRSEC, NSF-DMR-0081075 (AJM) and US-ONR (SDS) for support.

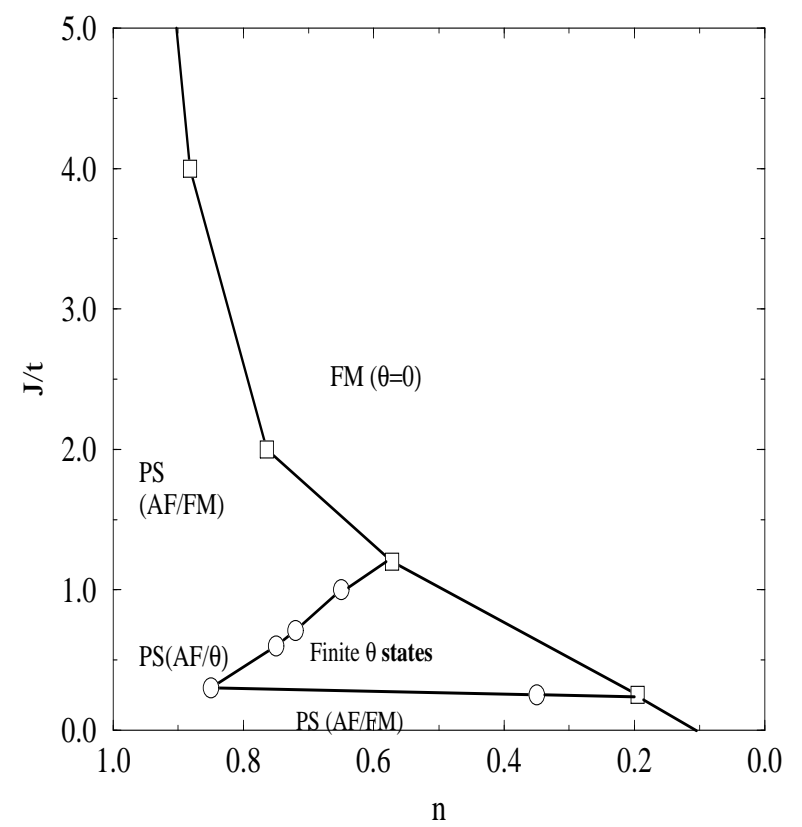

FIG. 1. The phase diagram of the double exchange model, as deduced from the analytic calculations of the free energy at all $J$.

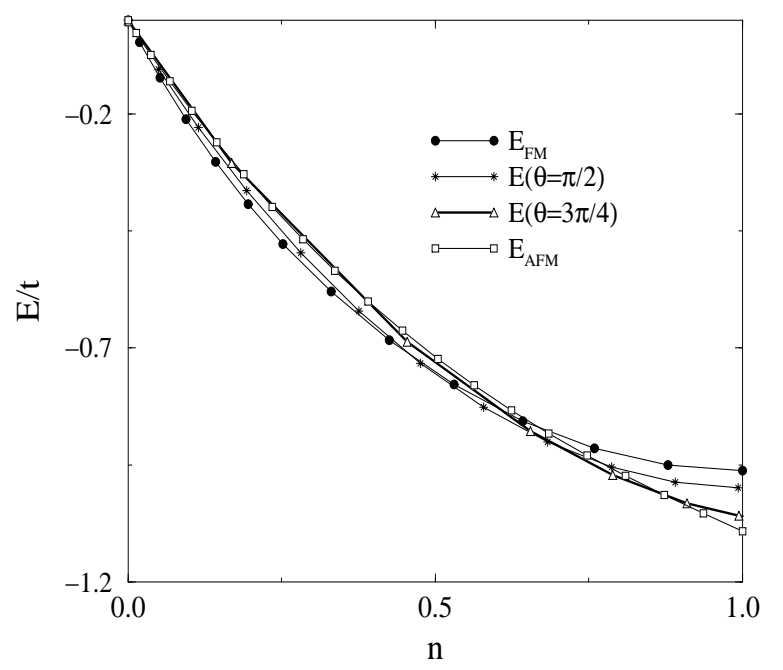

FIG. 2. Energy per orbital for $J / t=0.6$. There is a continuity of phases from $\theta=0$ to $\theta=3 \pi / 4$, followed by phase separation between $\theta=3 \pi / 4$ and $\theta=\pi$ 


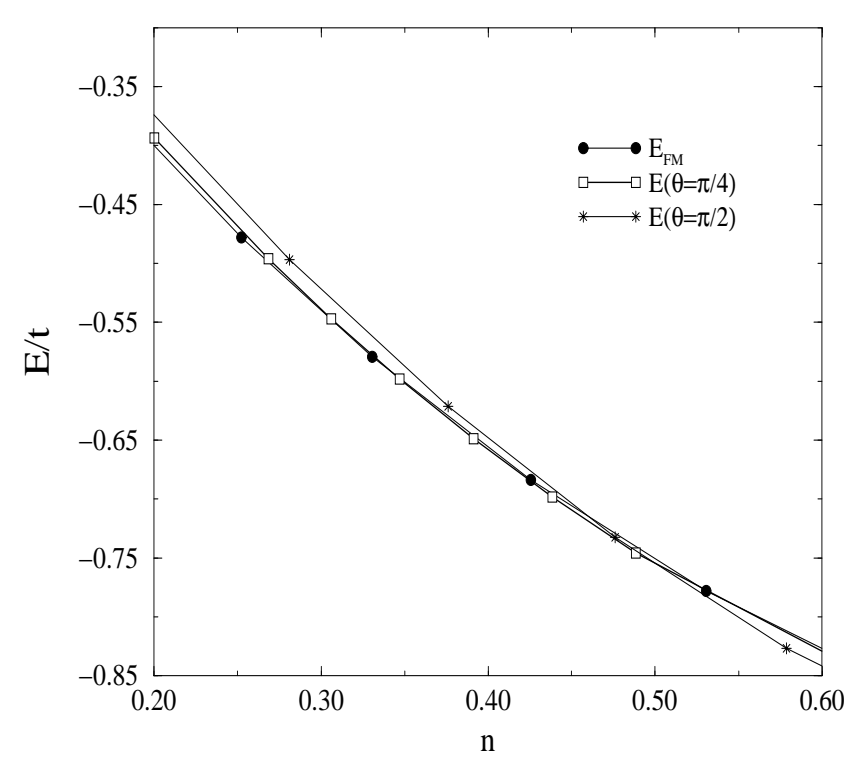

FIG. 3. The region between $0.2 \leq n \leq 0.6$ has been amplified to illustrate that the transitions between $\theta=0$ to $\theta=\pi / 2$ are second order. The second order transitions extend to the $\theta=3 \pi / 4$ state

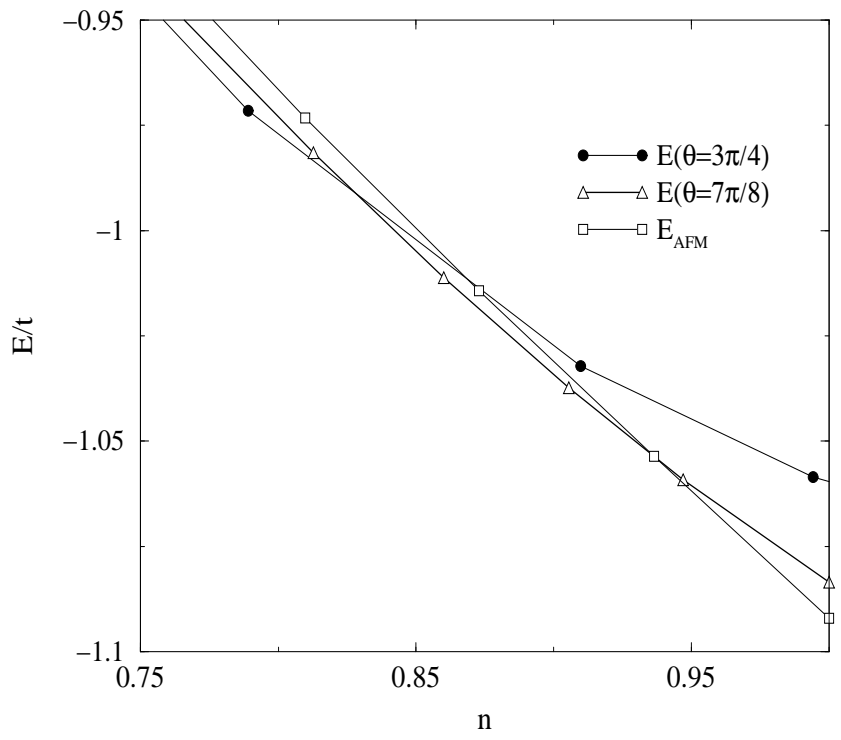

FIG. 4. The transition from the $\theta=3 \pi / 4$ state to the AFM state is first order, and the system phase separates.

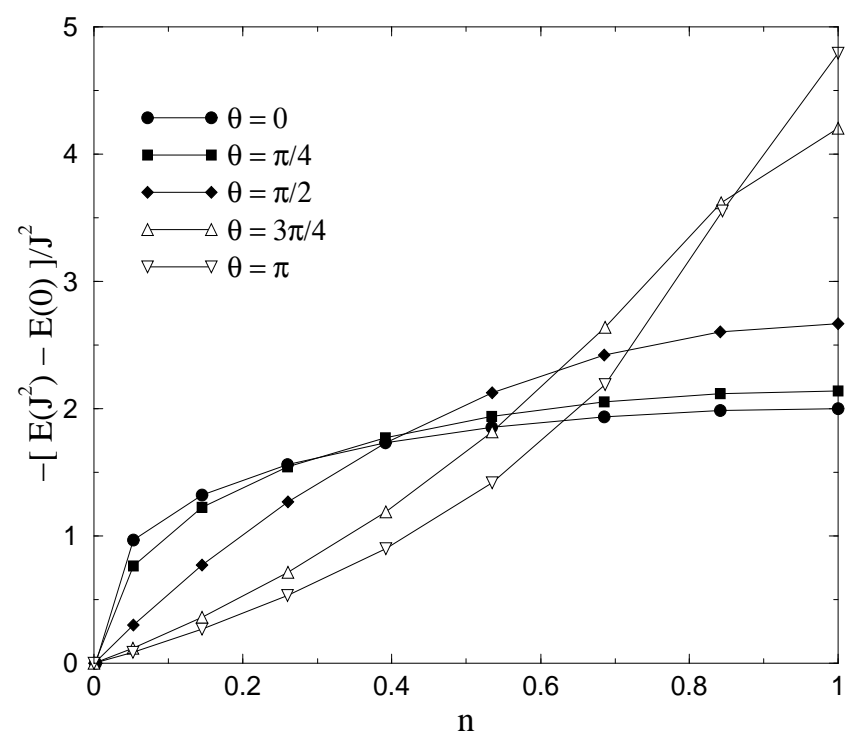

FIG. 5. Small $J$ energies of the states with different $\theta$ computed from Eqs. 7 and 8 at $J=0.05 t$. The wide regime of phase separation between $\theta=0, n=0.15$ and $\theta=\pi, n=1$ phases is evident.

${ }^{1}$ C. Zener, Phys. Rev. 82, 403 (1951); P. W. Anderson and H. Hasegawa, Phys. Rev. 100, 675 (1955); P-G. de Gennes, Phys. Rev. 118, 675(1960).

${ }^{2}$ For a recent summary of the status of the field see, e.g, Phil. Trans. Roy. Soc. A376, 1469-1712 (1998).

${ }^{3}$ H. Ohno, Science 281, 951(1998); S. Das Sarma, J. Fabian, X. D. Hu and I. Zutic, Solid State Communications (in press), and references therein.

${ }^{4}$ J. König, H. H. Lin and A. H. MacDonald, Phys. Rev. Lett. 84, 5628 (2000).

${ }^{5}$ A. Chattopadhyay, A. J. Millis and S. DasSarma, Phys. Rev. B. 61, 10738 (2000).

${ }^{6}$ N. Furukawa , J. Phys. Soc. Jpn. 63, 3214 (1994); N. Furukawa, J. Phys. Soc. Jpn. 64, 2754 (1995); N. Furukawa , in Physics of Manganites, ed. by T. A. Kaplan and S. D. Mahanti, (Kluwer Academic/Plenum Publ., New York, 1999).

${ }^{7}$ A. J. Millis, R. Mueller and Boris. I. Shraiman, Phys. Rev. B. 54, 5405(1996).

${ }^{8}$ K. Kubo and A. Ohata, J. Phys. Soc. Jpn 33, 21(1972).

${ }^{9}$ A. Georges, G. Kotliar, W. Krauth and M. J. Rozenberg, Rev. Mod. Phys 68, 13 (1996).

${ }^{10}$ A. J. Millis, B. I. Shraiman and R. Mueller, Phys. Rev. Lett. 77, 175 (1996).

${ }^{11}$ E. Dagotto, Science 283, 2034 (1999).

12 S. Yunoki, J. Hu, A. L. Malvezzi, A. Moreo, N. Furukawa and E. Dagotto, Phys. Rev. Lett. 80, 845(1998); E. Dagotto, S. Yunoki, A. L. Malvezzi, A. Moreo, J. Hu, S. Capponi, D. Poilblanc and N. Furukawa, Phys. Rev. B. 
58, 6414(1998).

${ }^{13}$ E. Mueller Hartmann, Z. Phys. B 74, 507 (1989); Z. Phys. B 76, 211 (1989).

${ }^{14}$ A. A. Abrikosov, L. P. Gorkov and I. E. Dzyaloshinski, Methods of Quantum Field Theory is Statistical Physics, Dover Publications (1963).

${ }^{15}$ A. Moreo, M. Mayr, A. Feiguin, S. Yunoki, E. Dagotto, Phys. Rev. Lett. 86, 135 (2001).

${ }^{16}$ J. L. Alonso, L. A. Fernandez, F. Guinea, V. Laliyena and V. Martin-Mayor, Phys. Rev. B 63, 064416 (2001). 